\title{
Rating of authors
}

\author{
Editorial
}

\section{Dear Readers,}

There is a vast theoretical background for evaluation of scientific works and there are a lot of ways how to, if possible, objectively evaluate the significance and quality of individual theses, authors and researches. Various evaluation elements are more or less objective in different branches of research and it is necessary to consider suitability of their use and "justness" of the final comparison. Then it depends on each metric, how it uses these and other parameters and how many iterations it logs. Elementary ways of calculation of these indicators of quality, their properties and scientific power evaluation of an researcher are briefly explained in this article.

\section{Citations}

One of the basic elements of quality and importance evaluation of the article are citations of given publication as well. In this case, with some exaggeration, the offer-demand system should work. Citations mean how many times the publication has been cited by other authors. In case of many citations, it is possible that the publication is very important for further study in the field of research.

The scientist is obliged to present all sources - the works of others he used. He should minimize the number of non constructive citations of authoritative or prestigious works. Often the syndrome of boss's citations can be seen - purpose of crediting authors with high academic degree, or citations of works written by editors and potential reviewers in order to increase the chances of the article for publication.

Special cases are autocitations. This term means correspondence between authors of cited and citing work. On average, these citations represent 20-30\% of the whole amount, databases like Web of Science are able to count citations with as well as without autocitations.

There is also the journal autocitation, that is when articles of the journal are cited, no matter who their author is. Not so clearly recognisable are cases when several groups of scientists make citations to help each other, co-workers from an institution or so called second generation cyclic citations - citing an article which is citing the writer's article.

\section{Cited half-life}

Cited half-life is a median of age of articles that were cited in the Journal Citation Reports (JCR) year. For example, if a journal has cited half-life 7 years, the number means that half of all cited articles were published more than 7 years ago and half less. This number can be used as an index of balanced quality of a journal.

\section{Altmetrics}

Altmetrics is acronym for alternative metrics, which is trying to consider all influence of science on society. Compared to indexes or citations, this metrics include not only articles, but conference presentations, posters, clinical studies, grants or social networks as well. Advantage of altmetrics is wider range of sources, disadvantage commercialisation and quality of data. 


\section{Eigenfactor score}

Eigenfactor project is an academic research project sponsored by University of Washington, which aims on using large network analysis for mapping structure of academic research. Compared to older methods, this project works on all stages of connection between articles, includes long-time period calculations of article's influence and takes into consideration also typical publication activity in relevant science branches.

\section{Number of articles}

Number of articles express how the author is active in publication work. Important thing is to express number of citations for past few years, usually for five. But for comparable result of all researchers is necessary to create indexes. Some of the most important are below in this article.

\section{Hirsch's index}

Hirsch index or H-index represents how many articles of the author reaches higher amount of citations than the serial number in a row of articles sorted by the number of citations. It is one of the citation indexes which describes response to an article published by an individual researcher

$$
h_{\text {index }}(f)=\max (i) \cdot \min (f(i), i) \cdot
$$

The index can be found for example on the Web of Science - section Citation report. It attempts to measure the productivity and an impact of a researcher in a single number. There are number of situations when H-index can be misleading. The main problem is, this index does not account typical number of citations and articles in different fields, but as was mentioned above, some branches are much more active in producing numbers of less significant results. It also does not take into account author's position in the list of writers, but after all the place is important in some disciplines.

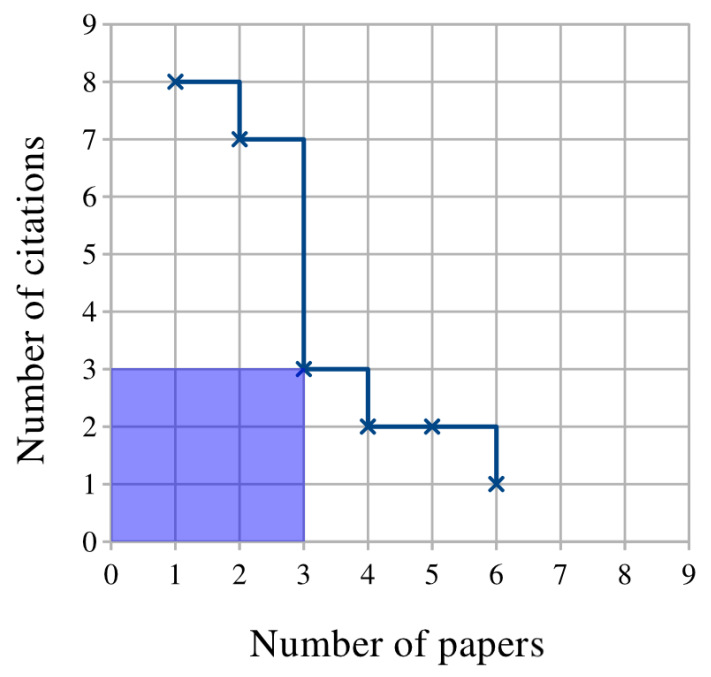

Figure 1: Principle of Hirsch's index 


\section{M-index}

The m-index is derived from Hirsch's h-index $(\mathrm{h})$ and depends on number of years $(\mathrm{n})$ between researcher's first and last publication. Formula for m-index is

$$
m=\frac{h}{n} .
$$

The m-index, also proposed by Hirsch, is defined as h-index divided by the number of years between the researcher's first and most recent publication. This allows comparison of early and late-stage scientists by introducing the correction time constant to the Hirsch's index. The m-index averages periods of high and low productivity throughout a career. Which may or may not reflect the current situation of the scientist, who can be in this time inactive in publication work, for example because of working on new methods, not having time or results to write about yet.

\section{iN-index}

iN index represents number of publications with at least $\mathrm{N}$ citations, usually featured with $\mathrm{N}=10$ or $\mathrm{N}=100$.

\section{G-index}

Let us have all author's articles in a row according to the number of citations they have, beginning from the highest. This index is the largest number such that the paper with the highest g-index have at least $\mathrm{g}^{2}$ citations. For example, if author's g-index is 10 , his best of the articles must have 100 citations.

\section{Tori-index}

The total research impact of a scholar (tori) is calculated using the reference lists of the citing papers (n), without self-citations of course. The addition of each citing article is normalized by the amount of the rest of references in the citing articles (r) and the number of authors in the cited work. In this formula, (a) stands for the number of members in a group of authors, (n) is the count of non-self citations mentioned in the article. The (r) is the number of resources which were used to write the descendant article, except the cited one. The tori-index is defined as the amount of work that others have devoted to the ones research, measured in research papers

$$
\text { tori }=\sum_{n} \frac{1}{a \cdot r}
$$

\section{Riq-index}

This abbreviation means Research impact quotient. The relationship between Riq-index and Tori index is analogous to the bond between $\mathrm{m}$ - and h-index. $\mathrm{h}$-index is in the formula replaced by the square root of the tori, $\mathrm{n}$ is number of years between first and most recent publication and the sum is multiplied by 1000

$$
r i q=1000 \frac{\sqrt{\text { tori }}}{n} \text {. }
$$

Geoinformatics FCE CTU 15(2), 2016 


\section{Conclusion}

Around the scientific world, different ways how to consider the quality of research and it's impact were created. Based on these results, grants politics or scientists migration works. Although even the best methods still can not compare absolutely objectively and complexly totally different branches, differences between states and departments. In general, for a scientist is important to publish in good reputed magazines and to be cited by other scientists eminent in their branch of research.

Veronika Stehlíková

Martin Urban

Ondřej Netvich

CTU in Prague - Faculty of Electrical Engineering 